\title{
Téoros
}

Revue de recherche en tourisme

\section{Enjeux stratégiques des parcs thématiques : Amérique du Nord et Europe}

\section{Michel Zins}

Volume 12, numéro 3, octobre 1993

Le renouveau des parcs à thèmes

URI : https://id.erudit.org/iderudit/1077930ar

DOI : https://doi.org/10.7202/1077930ar

Aller au sommaire du numéro

Éditeur(s)

Université du Québec à Montréal

ISSN

0712-8657 (imprimé)

1923-2705 (numérique)

Découvrir la revue

Citer cet article

Zins, M. (1993). Enjeux stratégiques des parcs thématiques : Amérique du Nord et Europe. Téoros, 12(3), 5-9. https://doi.org/10.7202/1077930ar d'utilisation que vous pouvez consulter en ligne.

https://apropos.erudit.org/fr/usagers/politique-dutilisation/ 


\title{
Enjeux stratégiques des parcs thématiques: Amérique du Nord et Europe
}

\author{
Michel Zins*
}

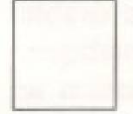

Formule magique du développement, de la gestion et du marketing touristique dans les années 1970 et 1980 , les parcs thématiques et leur devenir sont la grande énigme des années 1990 . Ceci est vrai non seulement pour les professionnels du tourisme et le grand public, mais également pour les gestionnaires mêmes de ces parcs.

Les raisons de ce revirement sont nombreuses. En premier lieu, les hésitations de l'empire Disney: les cachotteries sur les résultats récents aux États-Unis, le succès mitigé d'EuroDisney et le report, voire l'annulation du projet de Long Beach. Et quand Disney hésite, le reste de l'industrie panique. En second lieu, les difficultés que connaissent la plupart des autres parcs qui risquent de faire s'allonger la liste des fermetures et dépôts de bilan de parcs thématiques.

Il faut donc se pencher sur l'avenir des parcs thématiques, afin de comprendre les raisons profondes de leur succés original, de comprendre le malaise qu'ils connaissent aujourd'hui et d'identifier les enjeux devant lesquels ils se retrouvent, que leurs dirigeants en aient conscience ou non et qu'ils en acceptent l'évidence ou pas.

\section{L'impossible définition}

Une des premières difficultés rencontrées lorsque l'on veut se faire une idée des enjeux auxquels font face les parcs thématiquesest celle dela définition même de ce qu'est un parc thématique.

Plusieurs définitions sont offertes dans la littérature et les milieux professionnels. Inutiled'en faire la listeou la synthèse, mais toutes ces définitions ne recouvrent pas moins une réalité très variée. Ces variations quant au sens d'un parc à thèmes concernent :

- les thématiques proposées elles-mêmes;

Monsieur Michel Zins est president de Zins; Beauchesne et Associés et professeur à rUniversité Laval.
- la spécification de la thématique ou au contraire la largeur de l'éventail des thèmes abordés;

- le niveau d'activitéproposé auvisiteur ou requis par lui;

- l'aspect ludique;

- l'authenticité,

- la symbiose avec le milieu;

- la présence d'hébergement et l'aspect estation touristique (resort);

- l'intégration et l'auto-suffisance;

- la relation avec l'environnement;

- la taille.

Cette hétérogénéité de paramètres pour définir les parcs thématiques rend relativement complexe la compréhension des difficultés qui semblent les affecter ou au contraire des opportunités qui sont les leurs.

\section{À quelle époque appartient le parc thématique?}

Fin d'une épopée ou d'une époque, ou alors émergence d'une ère nouvelle, on ne sait trop à l'heure actuelle comment interpréter les remous qui caractérisent l'évolution récente des parcs thématiques.

L'expansion vertigineuse des parcs thématiques, en taille et en nombre, a débuté il y a plus de vingt ans déjà. Depuis, les deux porte-étendards qu'ont été le groupe Disney pour ce qui est des parcs thématiques «doux», principalement orientés sur la féerie, la famille, l'atmosphère et l'éducation, d'une part, et les Six Flags, pource qui est des parcs d'attraction *durs" (iron parks) dont la force de l'attrait réside dans la présence de manèges à sensations fortes, d'autre part, ont fait école. 
Mais ces parcs, vingt ans après, paraissent à certains égards (dans leur concept de base) sortir d'une autre époque ou appartenir à une autre génération. En effet, Disney comme son nom l'indique est issu d'un rêve de $W$ alt Disney et a bénéficié de toute la magie du monde merveilleux que cet homme avait créé et de l'immense outil promotionnel qu'est cet ensemble de films et de personnages. Les Six Flags, avec comme emblèmeMagicMountain, en plein désert non loin de Los Angeles, sont une fantastique fête foraine.

\section{Question d'envergure}

La taille, la superficie, le nombre d'attractions et la capacité d'accueil semblent tous être des éléments présentés comme des facteurs clés dans le succès des parcs thématiques, à tout le moins un critère de classification ou de mesure de leur succès. Mais qu'en est-il ?

Il est vrai que le gigantisme semble être relié au succès. Walt Disney avec ses milliards de dollars d'investissements, ses milliards de pieds carrés de parcs et autant en réserve foncière, ses 30 millions de visiteurs et 30 millions dedollars de budget publicitaire rien que pour le parc Walt Disney World près d'Orlando, estleleader incontesté de la catégorie. Six Flags et Anaheuser-Bush, avec ses Bush Gardens, semblent vouloir suivre, mais connaissent actuellement un certain essoufflement et une bonne dose d'inquiétudes et de déceptions.

De l'autre côté du spectre de la taille se situent de petits ensembles, souvent des parcs thématiques bien implantés régionalement ou avec une thématique précise, qui s"en tirent relativement bien. D'ailleursdansdenombreux parcs, la course vers le gigantisme, devenue trop périlleuse, a cédé la place, autant eu Europe qu'aux États-Unis, à un recentrage, voire une désescalade et un dếsinvestissement.

L'alternative semble donc être la suivante: soit on est un grand parc thématique d'envergure internationale et la masse critique devient très importante, près du milliard de dollars d'investissement, audessus de 10 millions de visiteurs et plus de 10 millions de dollars de publicité et promotion, ou alors on est un petit parc de moins de 1,5 million de visiteurs, implanté solidement sur un marché local ou sur un thème très spécialisé et positionné avec force grâce à un avantage concurrentiel majeur.

\section{Question de localisation}

Le débat reste ouvert d'autre part quant au choix de l'endroit qui est le plus favorable au développement d'un parc thématique. Derrière cette décision de localisation se profilent deux problématiques :

- lemilieu naturel d'un parcthématique est-il caractérisé par un climat chaud?

- un parc thématique doit-il se situer proche ou loin d'une grande métropole?

Bien évidemment, la première question a été vigoureusement soulevée à propos d'EuroDisney. N'aurait-il pas mieux valu installer ce parc quelque part en Europe méridionale où le soleil brillerait au-delà de 300 jours par année? Un climat froid et pluvieux n'encourage certainement pas la fréquentation d'un parc extérieur dont la majorité des activités ou du moins la plus grande superficie utile est en plein air. Rendre un parc utilisable à l'année longue ou le concevoir dans cette perspective en augmenterait assurémentlecoût. Ildevrait pouvoir alors entraîner une fréquentation accrue qui puisse permettre de couvrir les frais d'opération et de récupérer les investissements requis pour une opération annuelle.

Dans le cas contraire, l'établissement d'un parc thématique dans un climat moins clément en limite la fréquentation et la capacité d'autofinancement. Ellecontraint aussi ses promoteurs à concevoir un équipement moins coûteux nécessitant un achalandage moindre pour être rentable. Ou alors la thématique doit être adaptée à des conditions hivernales, favorisant l'utilisation et l'attrait du parcen hiver. Encore faut-il que celui-ci se situe alors dans des régions aux conditions hivernales et d'enneigement stables.

Pour ce qui est d'une métropole, c'est un faux problème. Le véritable enjeux c'est l'accès facile pour un nombre maximal de visiteurs potentiels.

\section{Question de thème et de positionnement}

La thématique fait partie de la définition mêmed'un parcthématique. Maisest-ce là sa véritable caractéristique distinctive? $\mathrm{Ou}$ réside-t-elle dans la notion de parc, ou peut-être ni dans l'une ni dans l'autre?

En fait de thème, les musées ou autres centres d'interprétation ne cèdent rien aux parcs thématiques. Etsil'image de ce qu'est le parc thématique du futur est illustré par un Epcot Center ou un Futuroscope, et que l'on considère les nouvelles orientations prises par les musées et centres d'interprétation, en particulier dans les domaines des sciences, de la biologie et de la technologie, on peut se questionner sur le positionnement relatif des deux institutions.

Pour ce qui est de la thématique, de nombreux enjeux doivent être pris en compte:

- Le renouvellement de la thématique elle-même ou du moins de son exposition ou de sa mise en oeuvre. Ceci est requis du fait de l'impact de plusieurs forces: l'évolution sociale et le passage des générations, l'évolution technologique, l'évolution de la thématique elle-même (le savoir qui la supporte pour ce qui est de l'histoire et de la biologie par exemple), les éléments de la thématique (personnages et acteurs pour des films; fusées, autos et avions pour les transports). Varient également le mode d'expression de la thématiqueou sa place dans la société.

- La concurrence accrue d'autres médias permettant l'accès facile et motivant à des thématiques (télévision, clubs vidé́o, ordinateurs, jeux électroniques) ou au jeu et à l'excitation (dans ce cas, on ajoutera à la liste précédente les arcades de jeux vidéo, les casinos, etc.).

- La concurrence indirecte des festivals, des centres de séjour et d'activités intégrés (Clubs Med et camps spécifiques pour les jeunes pour n'en citer que quelques-uns) qui viennent prendre leur part dans le temps et l'argent consacrés aux vacances et aux loisirs. 


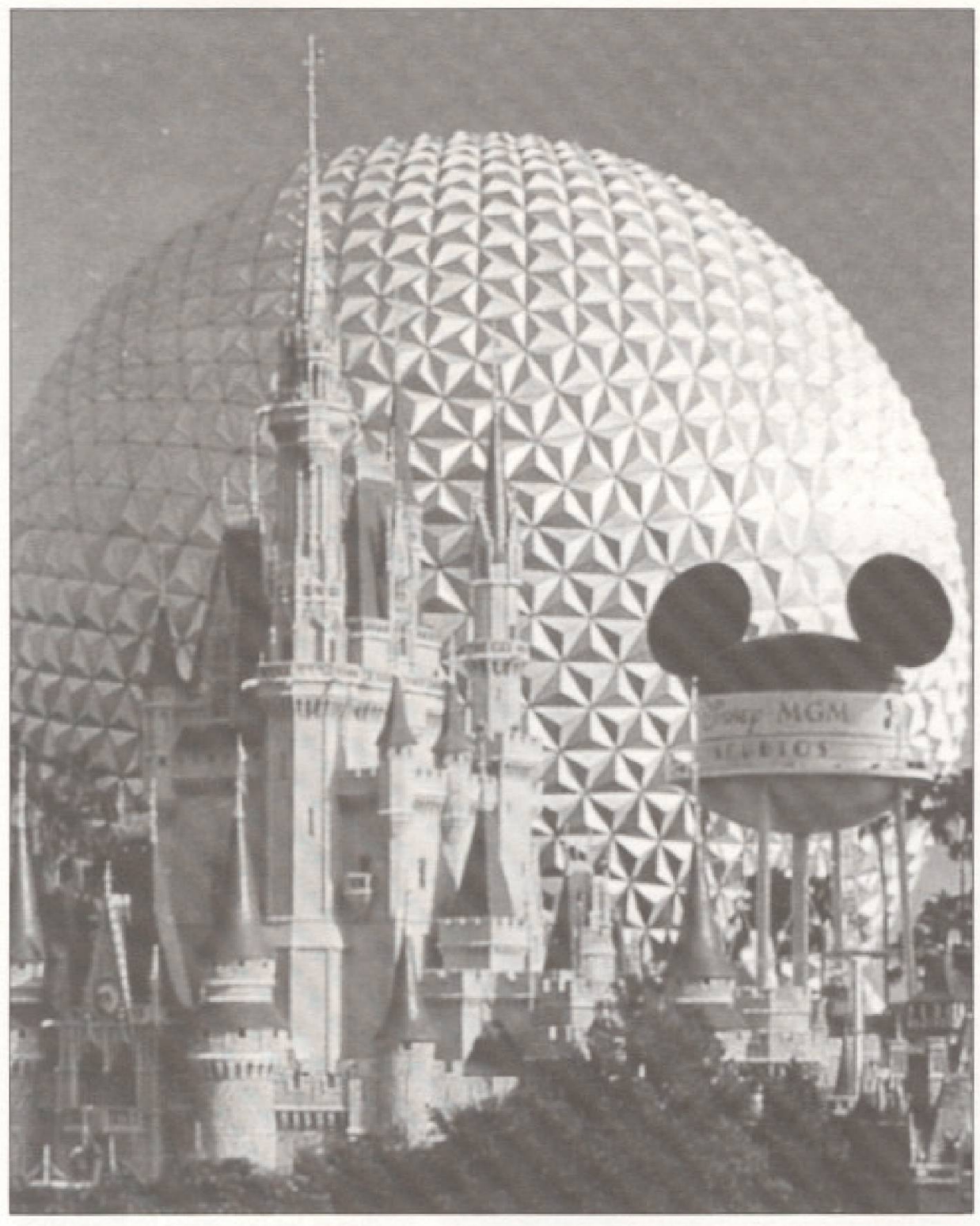

Dans un contexte où deplus en plus d'équipements, d'entreprises, d'institutions et de médias ciblent avec une précision et une force accrues les besoins, désirset fantaisies des jeunes et des moins jeunes, et ce, sur une variétéde thèmes, les parcs thématiques risquent de paraître comme des dinosaures d'un autre temps: grands, gros, inadaptés et incapables d'évoluer.

Les plus gros, sans doute, vont être capables de tirer profit de leur gigantisme mais au prix d'une promotion vigoureuse et d'une évolution constante. Walt Disney World d'ailleurs évolue vers un concept de *total destination resorto avec bien sûr le traditionnel Magic Kindgom, mais également Epcot Center, un centre d'activités nocturnes, des modes d'hébergements in-

\section{Un contexte changeant}

De toute évidence le contexte - ou dit de façon plus formelle le macro-environnement - dans lequel évoluent les parcs thérmatiquesachangé etcontinue d'évoluer de façon rapide. Où nous conduiront ces évolutions technologique, économique, culturelle et sociale? Nul ne le sait avec certitude. Maisd'ores et déjà les impacts de ces évolutions sur le futur des pares thématiques se font ressentir. Plus encore que ses effets, ce sont les questions soulevées qui sont importantes.

L'évlution tecbnologique représente le plus grand enjeu pour les parcs thématiques. Elle représente d'une part une menace certaine en créant une concurrence importante aux parcs par les possibilités d'éducation, d'expérimentation et de jeu qu'elles offrent mais aussi en accélérant l'obsolescence du produit offert et surtout de l'expérience proposée. Mais d'autre part, elle offre aussi une multitude d'opportunités permettant aux parcs thématiquesd'ameliorer ou de faire évoluer leur offre.

Les valeurs et l'environnement sacio-culturel sont également en pleine évolution. La sensibilité écologique et la montée de l'écotourimime sont de réelles menaces pour certains pares par les limites imposées à leur expansion maiségalement par l'intérêt croissant des gens pour les choses authentiques et reliées à la nature. Mais encore une fois il $\mathrm{y}$ a là une opportunité à saisir pour lesparcs thématiques ayant unevision et une bonne flexibilité stratégique. D'autres valeurs viennent également affecter l'avenir et le positionnement des parcs: la volonté d'une implication active des individus et d'une expression personnelle de ce qu'ils ressentent, le retour des activités parents-enfants mais dans une relation plus égale et respectueuse, l'exigence d'un ratio qualité/prix très bon et d'une qualité de service totale sans faille. périence pour le visiteur, ou alors en s'intégrant avec force sur un marché local ou régional. Entre les deux, l'échec est presque garanti.

Trouver un positionnement fort, appuyé sur un avantage concurrentiel défendu avec vigueur, en ciblant le bon marché: voilà une voie en dehors de laquelle les parcs thématiques, comme toutes les autres entreprises du monde, ne pourront espérer survivre.
Le contexte économique a bien changé aussi, la période de la dépense facile a cédé la place à la réalisation et à la priorisation des activités, des voyages et des achats. Cedi signifie pour les parcs une clientèle plus exigeante et plus difficile à attirer ou à satisfaireet à garder. De plus, la pression de la concurrence directe de la part d'autres parcs ou musées et indirecte de la part d'autres médias ou activités, ne fait que 
s'accroître sur la qualitéintrinsèque comme sur le rapport qualité/prix.

La démographie change également et aux années de la jeunesse dorée succèdent les années de la maturité argentée et éduquée. Pour répondreà ceschangements, les parcs doivent offrir un produit authentique, de qualitế et intelligent, mais également d'un accès aisé et offrant une atmosphère agréable, détendue et sécuritaire.

L'environnement politique et juridique a aussi évolué: la responsabilité envers les usagers et les employés, le respect de l'environnement, du développement durableetparfois, des politiques culturelles établissent un cadre de plus en plus contraignant pour le développement des parcs thématiques.

\section{L'expansion internationale des parcs thématiques}

Walt Disney World en Floride, en 1972, repousse les limites du succè̀ de son aîné Disneyland de Anaheim. Ceci devient encoreplusvrai avecl'ajoutd'Epcot Center et la constitution d'un véritable concept de séjour total (total destination resort). Six Flags et Anaheuser-Bush multiplient leurs parcs. De là à penser que comme pour McDonald's on a trouvé une formule magique que l'on peut transférer d'un continent à l'autre ou d'un pays à l'autre, il n'y a qu'un pas qui est facilement franchi. Et c'est un Walt Disney World qui est établi au Japon en 1983 et qui y remporte un certain succès. Suivra EuroDisney en 1992 qui suscite d'abord espoir etémerveillement à l'état de projet, mais qui très vite semble vouloir tourner au cauchemar ou du moins fait place au doute et à la déception. Les résultats de fréquentation sont là, mais le coeur n'y est pas ni dans le public, ni dans les communes et la région d'accueil, et surtout pas chez les gestionnaires. Et tout cela se déroule dans un contexte global de difficultés rencontrées par un grand nombre d'autres pares thématiques à travers le monde, et en particulier en France, où l'on a très vite voulu mettre en oeuvre cette formule magique.

Le contexte global et son importance pour un parc est particulièrement visible au niveau international. Le niveau d'évolution technologique, les facteurs socio-culturels, l'écologie, la situation économique, la démographie et le contexte politique, juridique et légal s'avèrent ainsi être autant d'éléments d'une extrême importance à prendre en compte lors du transfert international d'un concept de parc thématique.

\section{L'apport des parcs thématiques}

Perçu pendant un certain temps comme une solution miracle à toute épreuve, l'apport que représente le développement ou l'installation d'un parc thématique dans une région touristique semble vouloir être remis en question. Ses coûts et inconvénients apparaissent d'ailleurs de façon plus aiguë à la lumière des récents déboires connus par beaucoup d'entre eux.

L'apport en termes de retombées économiques directes pour le milieu environnant est souvent contesté, après avoir été affirmé. Walt Disney World a bénéficié à Orlando parce que cette dernière a su en profiter en s'organisant de façon dynamique pour le marché des congrès. Par contre, les municipalités autour d'EuroDisney contestent en grande partie son apport. Le problème en termes de retombées est que ces pares, quand ils sont importants, sont souvent intégrés et ont leurs propres services d'hébergement et de restauration. Ils n'intègrent presque pas le milieu environnant. Les emplois sont pour la plupart mal rémunérés et précaires.

Les inconvénientsperçus sontqueces parcs se «plaquents sur la région sans s'y fondre. Ils se servent de la région comme aterrain d'établissement» mais n'y apportent que peu en termes de valorisation culturelle voire touristique. Au fond, souvent les parcs thématiques traditionnels pourraient être situés *n'importe où». La clientèle touristique qu'ils attirent ne s'intéresse pas facilement à la région avoisinante etn'est là que pour le parc lui-même.

Finalement, quand ils sont importants, les parcs drainent un flux considérable de touristes lors des périodes de pointe avec tous les inconvénients que cela représente en termes de trafic, de pollution, de bruit et de changements pour les résidents locaux.

\section{Un diagnostic inquiétant}

Le diagnostic que l'on peut poser sur les parcs thématiques est donc quelque peu inquiétant: même si de nombreuses opportunités s'offrent à eux, des menaces certainespèsent quand mêmesur la plupart des parcs et sur le concept de parc luimême. Des faiblesses et contraintes leurs sont inhérentes, mais ils ont des atouts importants à jouer pour la plupart. On peut ainsi dresser le tableau suivant :

\section{Opportunités}

- la curiosité et l'in térêt grandissant de la population et des touristes pour une variété de thèmes;

- le niveau d'éducation plus grand de la population;

- les possibilités technologiques pour les présentations et les activités;

- le temps de loisirs disponible;

- la priorisation des loisirs et del'éducation;

- la volonté croissante de passer des séjours familiaux (parents-enfants) enrichissants;

- la recherche d'expériences et de séjours intégrés;

- la croissance des préférences pour des séjours courts.

\section{Menaces}

- la concurrence croissante de «nouveaux muséesw;

- la concurrence des alternatives de loisirs et d'éducation par les nouveaux médias et les nouvelles technologies;

- le resserrement des budgets familiaux pour les loisirs;

- la recherche d'expériences authentiques;

- l'évolution de l'écotourisme;

- l'obsolescence des équipements et la perte d'intérêt pour la thématique.

\section{Atouts que les parss peuvent jouer}

- l'approfondissement d'une thématique ou d'une expérience;

- l'intégration de l'expérience;

- l'accessibilité à la famille; 
- la qualité totale du service par une gestion intégrée;

- la force d'impact sur le marché.

Faiblesses et contraintes

que les parss peuvent connatre

- le manque de flexibilité́;

- l'étroitesse ou la confusion de la thématique;

- la non pertinence ou le manque d'attrait de la thématique;

- la difficulté d'accès;

- les files d'attente;

- la surfréquentation en période de pointe;

- le dépassement technologique;

- l'obsolescence de la thématique.

\section{Les enjeux globaux}

Au-delà des éléments clés qui vont déterminer le succès ou non d'un parc thématique, l'ensemble des parcs thématiques font face pour la prochaine décennie à un certain nombre d'enjeux majeurs quevoic:

- Lepositionnement, etvoire La raisond'etre, du parc thématique. Ceci est sans doute, face à l'evolution des musées, des centres de séjour de vacances, et des nouvelles technologies de l'information et de l'image, y compris les simulateurs appliqués à l'apprentissage ou au jeu, l'enjeu principal.

- Le choir du theme, et surtout le spectre couvert par celui-ci. Ceci représente un autre enjeu, qui va de la spécialisation extrême sur un personnage, un jeu, une technologie, une coutume ou autre, jusqu'à un concept global de centredeséjour total (totaldestination resort) vers lequel tend Walt Disney World. Le complexe de Floride propose ainsi le plaisir à toute la famille et à tout le monde et combine Mickey Mouse, la gastronomie internationale, les télécommunications, les sciences de la terre, la musique country et disco, le golf, les beauxhôtels, le ciné-studio, les salles de conférence et de congrès... et ce n'est qu'un début, paraît-il, étant donné la vaste réserve foncière de Buena Vista.

- Le renouvellement de l'affre.

- Le respect de la recherche d'expériences autbentiques, par un towriste actif, éduqué et soucieux de l'environnement, voulant emmener sa progéniture avec lui, aux goûts difficiles, tout en étant soucienux d"obtenir une bonne valeur pour son dollar.

- Le respectetl'intégration du milieu emvironnement.

- Lagestion despointes de fréquentation, en maintenant une qualité des services sans faille, et en évitant les files d'attente et l'engorgement, car il est dit que «trop de foule et trop d'attente tuent le plaisir».

- La gotion de la saisonnalité.

- Le maintien de la rentabilité.

\section{Des facteurs clés de succès}

Pour qu'un parc thématique soit capable de survivre et d'être un succès, celui-ci doit réussir à répondre de façon efficace à un certain nombre de critères ou de facteurs qui sont à la base de son succès. Les éléments les plus déterminants sont ainsi:

- une thématique forte et claire (si possible unique pour le marché visé);

- un marché et une clientèle bien définis qui soient capables de générer la fréquentation requise;

- une facilité d'accès au parc pour cette clientèle, appuyée sur un choix judicieux du site;

- un budget promotionnel adéquat;

- une qualité de service sans faille, et du niveau requis par la clientèle visée et l'expérience proposée;

- une définition claire et précise de l'expérience proposée au visiteur;

- une bonne gestion des files d'attente et de la foule lors des pointes de fréquentation;
- une stratégie de gestion de la saisonnalité;

- le renouvellement constant pour rester en phase avec l'évolution de la thématique, s'adapter à l'évolution technologique ou à une éventuelle baisse d'intérêt pour le thème ou son mode de présentation;

- une gestion serrée des coûts d'opération et une rentabilité permettant d'autofinancer le renouvellement des équipements;

- un financement adéquat permettant une marge de manoeuvre pour l'adaptation du parc.

\section{Conclusion}

Les parcs thématiques évoluent comme tous les autres équipements dans uneèrede turbulence importante due à l'évolution simultanée de la technologie, du contexte culturel et social et de la situation économique et démographique.

Face à des enjeux majeurs, ils se doivent, pour survivre, d'adopter un mode de gestion stratégique qui est loin de l'apparente facilité du succès de quelques-uns de leurs leaders.

Ils font l'objet d'une remise en question et d'une concurrence acharnée venant de toutes parts. Seule une réflexion sur leur thématique, l'expérience proposée et les clientèles visées leur permettra de se maintenir, pour certains, ou de se tailler enfin, pour d'autres, en difficultéou en gestation, une place dans leur marché. $f$ 